\title{
Percutaneous Endoscopic Gastrostomy Tube Placement: A Single Center Experience
}

\author{
Ankur Gupta ${ }^{1}$ Anil K. Singh ${ }^{2}$ Deepak Goel ${ }^{3}$ \\ ${ }^{1}$ Department of Gastroenterology, Max Hospital, Dehradun, \\ Uttarakhand, India \\ ${ }^{2}$ Department of Neurosurgery, Max Hospital, Dehradun, \\ Uttarakhand, India \\ ${ }^{3}$ Department of Neurology, Max Hospital, Dehradun, Uttarakhand, \\ India \\ ${ }^{4}$ Department of Oncosurgery, Max Hospital, Dehradun, \\ Uttarakhand, India \\ ${ }^{5}$ Department of Radiology, Max Hospital, Dehradun, Uttarakhand, \\ India
} Akash N. Gaind $^{4} \quad$ Shireesh Mittal ${ }^{5}$

J Digest Endosc 2019;10:150-154

\begin{abstract}
Address for correspondence Dr. Ankur Gupta, Department of Gastroenterology, Sahara Hospital, Viraj Khand, Gomti Nagar, Lucknow 226010, India (e-mail: mail.guptaankur@gmail.com).
\end{abstract}

\begin{abstract}
Keywords

- endoscopy

- gastrostomy

- postoperative

complications

- peritonitis

- enteral nutrition

Introduction Percutaneous endoscopic gastrostomy (PEG) tube placement is one of the recommended methods for providing enteral feeding in patients with swallowing difficulty and intact gastrointestinal tract. We review our three years of experience pertaining to PEG placement in our hospital.

Methods Records of all the patients, who underwent PEG between May 2014 to September 2017, were reviewed and relevant clinical and procedural details were noted. For all the patients, the procedure was conducted under antibiotic prophylaxis, moderate sedation, and local anesthesia. The PEG tube was placed by the "pull up" method. Telephonic follow-up of the patients was carried out after one month of study completion.

Results The PEG tube was placed in 73 patients (male 51 [69.9\%]; age median [range] 67 [16-91] years). PEG was placed in 42 patients with stroke (57.6\%), other neurologic disorders $17(23.3 \%)$, coma due to head injury 5 (6.8\%), and terminal malignancy 9 (12.3\%). Technical success was achieved in 73 (97\%) patients. Eleven procedurerelated complications occurred in nine patients (15.5\%) including one death due to peritonitis. Of the 57 patients, who could be followed-up after discharge, 41 died of their primary illness after $65(1-751)$ days, nine were alive and continuing on PEG tube feed, and in seven PEG was removed because it was not needed.

Conclusion PEG is a useful procedure for enteral feeding. Although procedural success is high, it may be accompanied by significant complications.
\end{abstract}

\section{Introduction}

Nutrition is essential for perpetuation of life. In patients with functionally intact bowel, enteral nutrition is always preferred over parenteral nutrition, especially if needed for longer duration. If normal swallowing is impaired, enteral nutrition could be provided through tube feeding which is passed through the nostrils (nasogastric or nasoduodenal tubes) or percutaneously. Tube feeding is accepted as a temporary method of feeding for the patients with acute illnesses who need support for a few weeks. Nutrition in a chronically debilitated patient, who requires long-term support, is preferably provided by percutaneous methods. Percutaneous access to the bowel can be achieved either surgically or endoscopically.

The percutaneous endoscopic gastrostomy (PEG) tube is a method used to achieve access to bowel and placed with the help of esophagogastroscope. Our center is a tertiary care 
referral center serving a large number of patients with neurological illnesses and terminal malignancy. We place the PEG tube frequently in order to provide nutrition to such patients. Here, we review our experience of PEG tubes placed over a three-year duration.

\section{Methods}

We retrospectively reviewed the care records of all those patients in whom PEG tube was placed between April 2014 and September 2017. All the patients were identified by reviewing endoscopy records. Relevant clinical and procedural details of every identified individual patient were recorded in a predesigned data collection form.

The PEG tube placement procedures were conducted after hospitalization and necessary investigations. All PEG tubes were placed by single endoscopist and assisted by the same nurse. Necessary investigations such as hemogram and prothrombin time were carried out; it should preferably be done within 24 hours prior to the procedure. Antiplatelet agents were withheld for 3 days and anticoagulant agents for 5 days before procedure for patients already on such drugs. As a part of routine medical care, explained written and verbal consents for procedure were taken from the patients or their close kin.

We placed 24 French PEG tubes (Boston Scientific, 24Fr Standard PEG Kit, Pull) in all patients. The pull method was used in all patients (-Fig. 1A-E). ${ }^{1,2}$
To provide standard of care, we administered prophylactic antibiotic (Ceftriaxone $1 \mathrm{~g}$ intravenous 30 minutes before the procedure) in all patients. ${ }^{3,4}$

The procedure was conducted after a minimum of 6 hours of fasting and under sedation administered by trained anesthetists. Feeding was started after 8 hours of PEG tube placement and patients were discharged soon if the feed were tolerated well; hospitalization was extended if required. After one month of completing this study, patients were telephonically followed-up and outcomes were assessed in the form of death from primary disease, and ongoing feeding with PEG or removed PEG (for those who could resume oral feed).

\section{Results}

Clinical and demographic details of the included patients is summarized in - Table 1. Over the study duration, 76 patients were referred for PEG; it was contraindicated in one patient with malignant ascites. A significant percentage of patients $(69.9 \%, 51$ of 73$)$ were male. The median age of patients was 67 and the range was between 16 to 91 years.

Technical success was achieved in 73 of 75 patients (97.3\%) patients. Complications occurred in 9 of 73 (12.3\%) patients, including the death of one patient from peritonitis. Two of the patients experienced two complications each. In one of the patients, it occurred in the form of infection on day three when he improved; later, he had inadvertently removed the
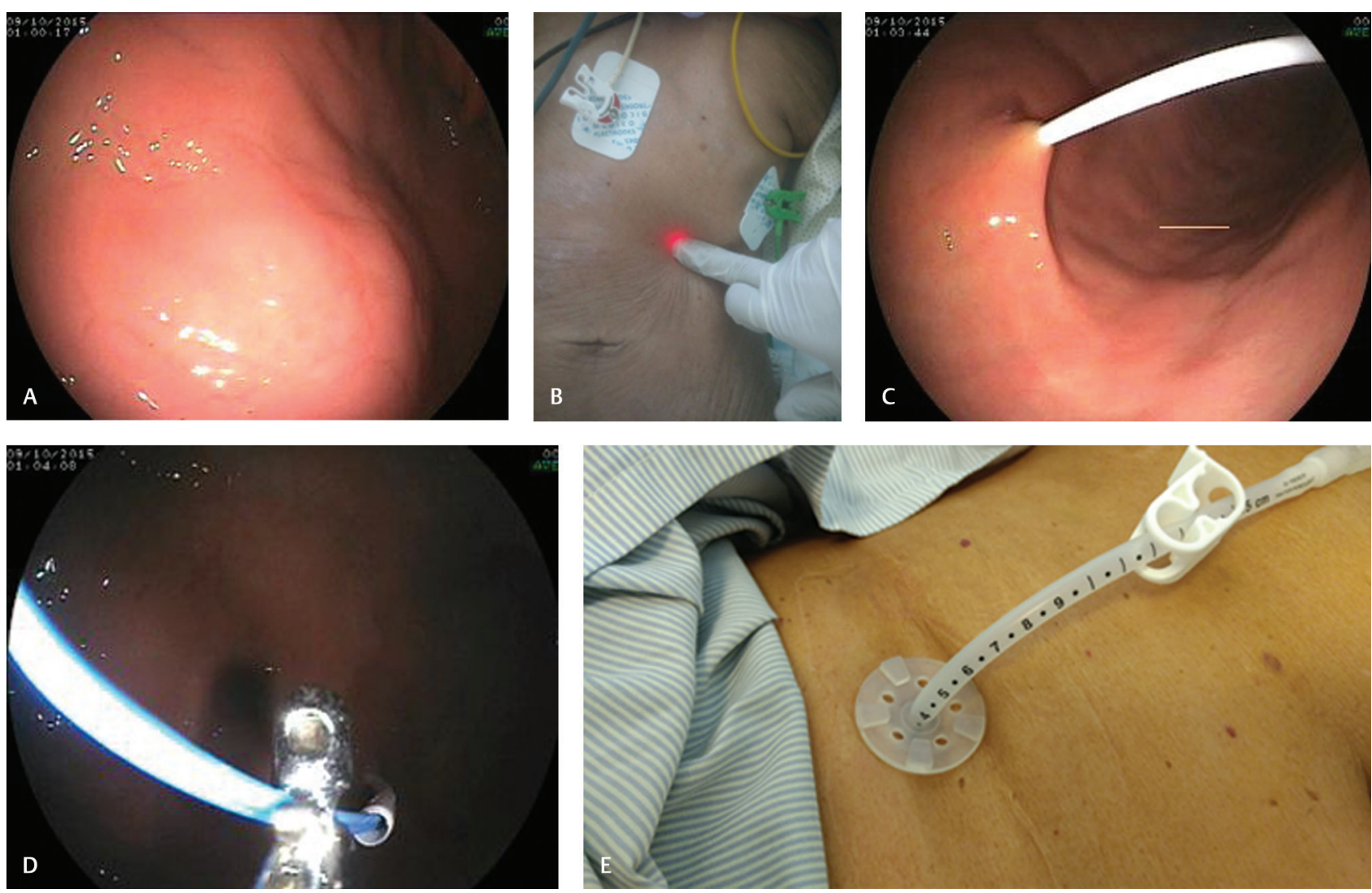

Fig. 1 Finger indentation after gastric insufflation. (b) Illumination may be visible transcutaneously in patients of thin build. (c) Needle with sheath entering the gastric lumen after piercing the abdominal and gastric wall. (d) Wire being held by forceps (or snare) to be pulled orally. (e) A PEG tube placed in a patient. PEG, percutaneous endoscopic gastrostomy. 
Table 1 Clinical characteristics of the included patients and relevant procedural details $(n=73)$

\begin{tabular}{|l|l|l|}
\hline Variable & \multicolumn{2}{|c|}{ Value } \\
\hline Age (years) & 67 & $(16-91)$ \\
\hline Gender & \multicolumn{2}{|l|}{} \\
\hline Male & 51 & 70 \\
\hline Female & 22 & 30 \\
\hline $\begin{array}{l}\text { Basic disease requiring } \\
\text { gastrostomy }\end{array}$ & \multicolumn{2}{|l|}{} \\
\hline Stroke & 42 & 58 \\
\hline Other neurological illness & 17 & 23 \\
\hline Terminal malignancy & 9 & 12 \\
\hline Head injury with coma & 5 & 7 \\
\hline Complications & \multicolumn{2}{|}{} \\
\hline Infection & $5 a$ & $3^{\text {a,b }}$ \\
\hline Inadvertent tube removal & 1 \\
\hline Respiratory depression & $1^{\dagger}$ & 1 \\
\hline Pneumoperitoneum & & \\
\hline Death due to peritonitis & & \\
\hline
\end{tabular}

Numerical data are expressed as either median (range) or percentage as appropriate.

${ }^{a}$ One patient developed infection and later had inadvertent tube removal.

'One patient developed inadvertent tube removal followed by pneumoperitoneum.

tube, which was managed with insertion of new PEG from the same fistulous opening. In another patient, pneumoperitoneum developed during the attempt to reinsert PEG endoscopically after he had inadvertently removed the PEG tube ( - Table $\mathbf{1}$ ).

\section{Complications \\ Infection}

Infection of site was the most common complication. It occurred in five patients. In two of them, it occurred on day 3 with pus formation and erythema developing during the hospital stay for their primary illness. Both of these patients were running a fever; in one of them, the pus culture grew Klebsiella pneumonie and the other showed the growth of

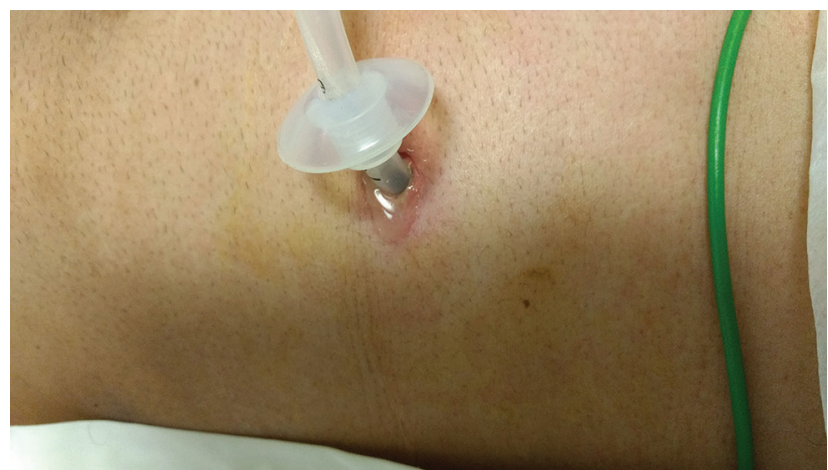

Fig. 2 Pus seen at the PEG site. PEG, percutaneous endoscopic gastrostomy.
Eschereschia coli. In the former patient, it was the same organism as the culture was revealed from other sites $(\boldsymbol{-}$ Fig. 2 ). The latter patient had urinary tract infection, which was treated simultaneously. In both of them, the infection improved with local antiseptic dressing and culture-based intravenous antibiotics by day five.

The third patient, in whom caretakers omitted dressing after discharge, presented after two weeks of PEG insertion and had abscess tracking from the PEG site (-Fig. 3). Pus culture showed Eschereschia coli. He required removal of PEG tube and incision drainage with culture-based antibiotics which was managed with nasoduodenal tube feeding.

The fourth and fifth patients had mild infections which occurred after one month (Klebsiella pneumonie) and two months (Pseudomonas aeruginosa), respectively, and controlled with local measures.

\section{Inadvertent Removal of PEG}

Three patients suffered inadvertent removal of PEG tube; all of them were neurologically compromised and agitated. One patient pulled the tube after 8 weeks of placement and reported to the hospital within 5 hours of the event; the PEG tube could be successfully placed from the fistulous tract made during the earlier procedure $(-$ Fig. 4$)$. The second patient, with initial PEG placed a year ago and replaced

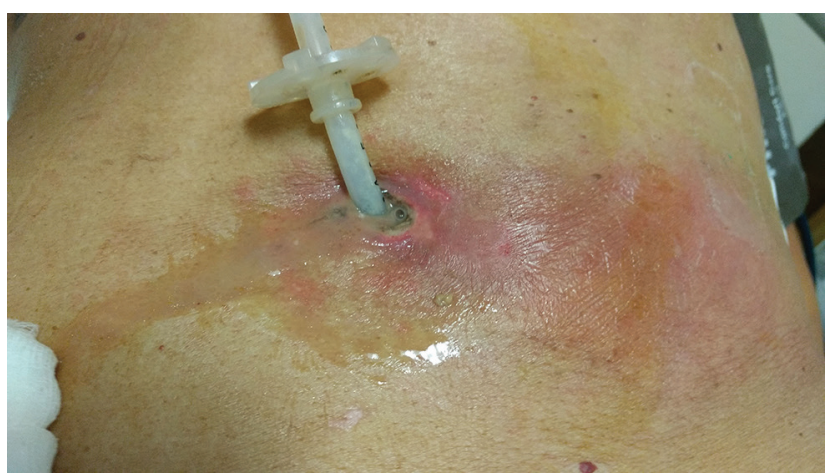

Fig. 3 Abscess tracking from the peristomal site.

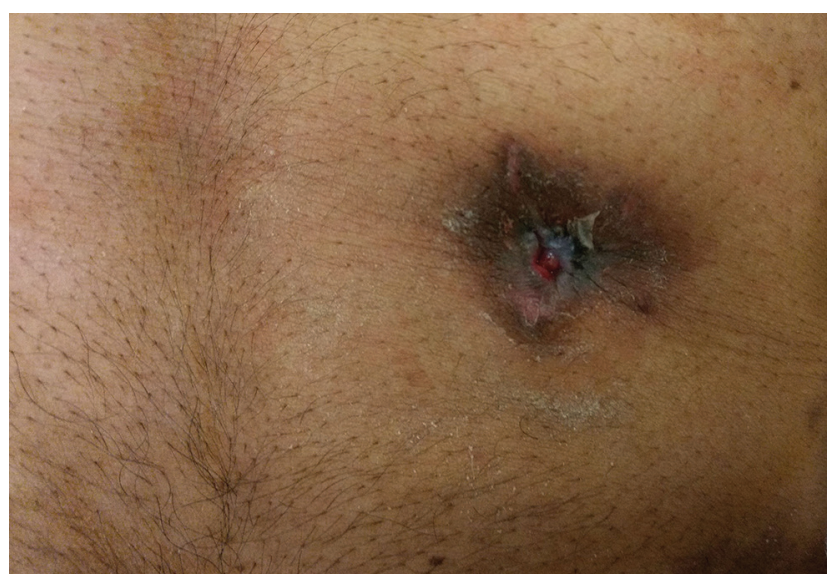

Fig. 4 Fistulous tract of an accidentally pulled out PEG tube, new tube could be placed through the same tract. PEG, percutaneous endoscopic gastrostomy. 
8 weeks ago, presented after 24 hours of removal and a repeat PEG had to be done with a fresh puncture. The third patient developed pneumoperitoneum which is described here.

\section{Anesthesia Related Complication}

During PEG placement, an 85-year-old female, with upper esophageal malignancy, developed respiratory depression with fentanyl, which improved with bag-valve-mask (BVM) ventilation. The procedure was completed successfully after a few minutes when the patient improved.

\section{Pneumoperitoneum}

One of the agitated patients pulled out the PEG tube within 24 hours of placement, and a repeat attempt to replace PEG was made after 48 hours of the event. The patient developed abdominal distension and the procedure was abandoned; on CT scan, the patient was confirmed to have developed pneumoperitoneum. Due to persistent distension, the patient underwent laparotomy with closure of PEG site and feeding jejunostomy (FJ) placement improved.

\section{Peritonitis}

One patient with carcinoma larynx had major complication in the form of peritonitis and succumbed despite surgical intervention. He developed abdominal pain after 24 hours of PEG placement and abdominal CT confirmed free peritoneal air and perforation peritonitis (-Fig. 5). He was initially managed with radiologic drains and later with laparotomy, when he developed septic shock and could not survive and died on day 7 of the procedure.

\section{Follow up}

A significant percentage of patients ( $79.5 \%, 58$ of 73 ) could be followed-up after one month of completing study (-Table 2 ), while 41 patients had died from their primary disease after a median time of 65 days with a range from 1 to 751 days. Nine patients were alive and on PEG feeds, and seven patients had PEG removed and were accepting oral feeds. One patient had died due to peritonitis at day 7 of the procedure.

\section{Discussion}

The PEG tube has several advantages over nasogastric or nasojejunal tubes. Besides the potential to reduce discomfort, PEG tube also avoids the complications associated with nasogastric tube, such as aspiration and gastroesophageal reflux. Moreover, PEG may be socially more acceptable as compared with nasogastric tube and has been shown to be nutritionally more efficacious. ${ }^{5,6}$ Ever since the innovative method of PEG was described in 1980 by Gauderer et al, this method has been extensively used in patients with patent gastrointestinal tract, who need enteral feeding for long duration. ${ }^{1}$

Technical success of PEG is high in our series, as it could be successfully performed in $97 \%$ of patients. In two patients, transillumination could not be achieved and, hence, the procedure could not be conducted. As expected, a majority of the

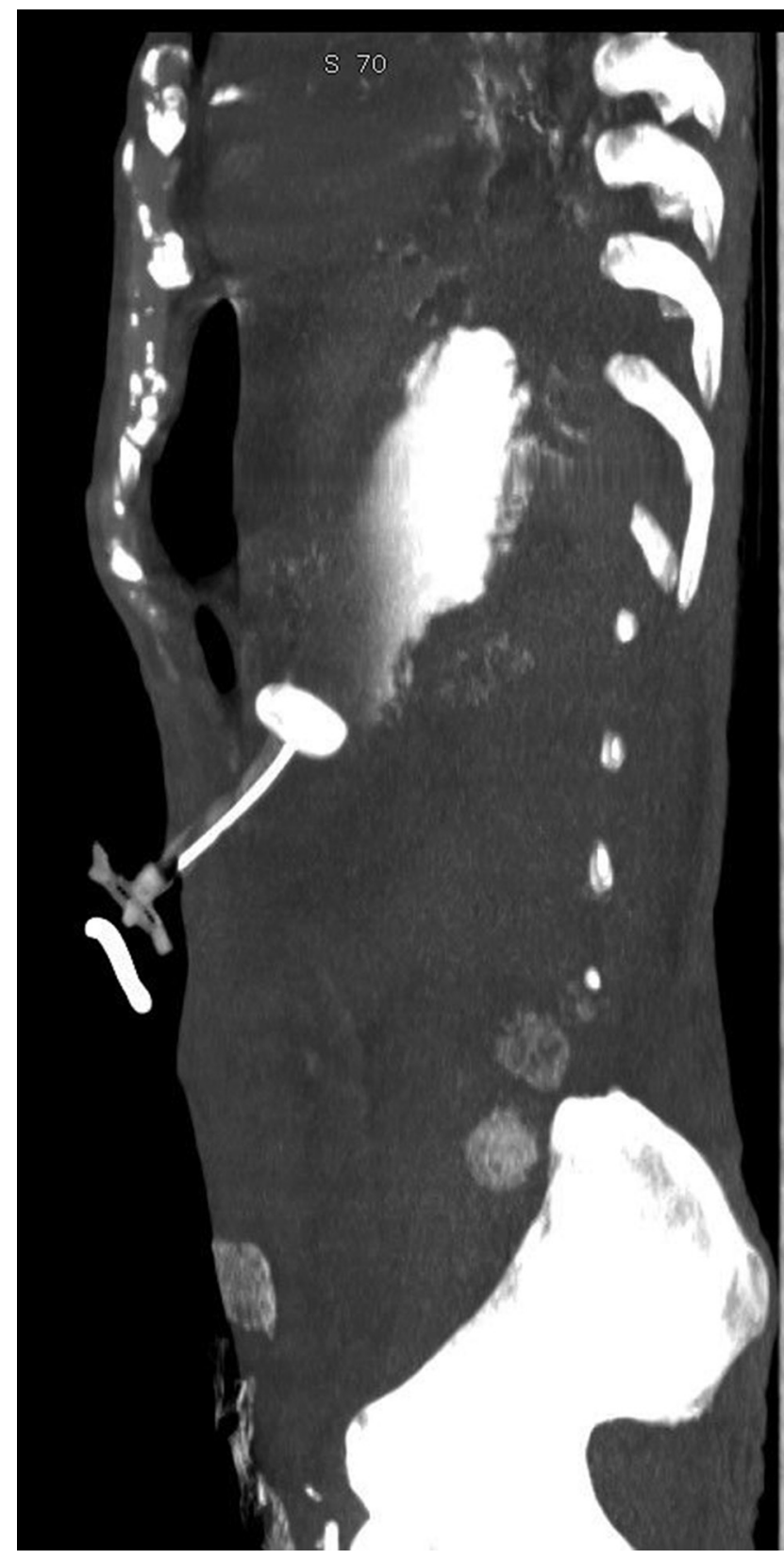

Fig. 5 Sagittal section of CT scan showing PEG tube in gastric lumen and pneumoperitoneum. PEG, percutaneous endoscopic gastrostomy.

Table 2 Follow-up data after one month of study completion

\begin{tabular}{|l|l|}
\hline $\begin{array}{l}\text { Follow-up data available, } \\
\text { number (\%) }\end{array}$ & $58 / 73$ (79.5\%) \\
\hline Details, number & 41 \\
\hline Death due to primary disease & 9 \\
\hline PEG removed & 7 \\
\hline On PEG feeds till last follow-up & $1^{\text {a }}$ \\
\hline $\begin{array}{l}\text { Death due to PEG related } \\
\text { complication }\end{array}$ & \\
\hline
\end{tabular}

Abbreviation: PEG, percutaneous endoscopic gastrostomy.

aThis is the patient who died due to peritonitis as a complication. 
patients were elderly, with the median age being 64 years. The youngest one (16 years) had tuberculous meningitis and the oldest one (91 years) suffered a stroke. Stroke was the commonest indication for PEG and was the reason for obtaining enteral feeding access in $55 \%$ of patients.

Infection of the PEG site was the most common, with complications occurring in five patients. While the antibiotic prophylaxis and optimal sterile dressings reduce the rates of infection, it remains the commonest reported complication. In our series, five patients developed infections, of which one was with pseudomonas and the others were with coliforms; all were monomicrobial. One patient had severe infection with subcutaneous abscess; in this patient, PEG had to be removed and incision drainage was carried out for the abscess. All other patients had minor infections which were controlled with antibiotics and meticulous dressing of the PEG site.

Three of our patients had accidentally removed the PEG; all were neurologically compromised and agitated. In one of the patients who presented early, PEG could be placed from the same site, while in the other who presented after 24 hours of PEG removal, the procedure had to be done afresh, since the external orifice had started to close. We suggest hand mittens as a simple precaution for the patients who are agitated and unable to avoid accidental removal of PEG tubes. In the third patient, in whom PEG was pulled out early and an early attempt was made to replace the PEG, pneumoperitoneum developed; he improved after being managed surgically. If the PEG tube is accidentally removed, we suggest that a new attempt be made after a week if the initial PEG placement happened less than a week ago. In case the initial PEG was placed more than a week back, fistulous tract is mature; hence, a PEG placement from the same site should be tried as soon as possible, preferably within few hours of the event to avoid closure of the site. In case a PEG replacement is tried after 24 hours of the tube removal, the tract is likely to be closed and procedure needs to be done as a fresh one. ${ }^{7}$

Perforation peritonitis is the most feared complication of PEG; although uncommon and despite surgical management, it has a high-rate of mortality. It is important to look for peritonitis specifically among obtunded patients, who develop subtle signs, due to their inability to communicate. Our patient with perforation peritonitis had laryngeal carcinoma, and he developed abdominal pain after 24 hours of PEG placement. He could not survive despite surgical intervention. Patients should be carefully monitored after the first feed to look for potentially fatal complications like peritonitis. In these patients, who are often neurologically compromised, symptoms of complications may be minimal; hence, the threshold of investigating the patient should be low. Although pneumoperitoneum after PEG is not uncommon, presence of clinical signs should prompt evaluation for peritonitis. ${ }^{8}$

In a study of 260 patients, Mansoor et al reported complications in $25 \%$ of their patients who underwent PEG for head and neck malignancy. In this study, 10 patients developed major complications and three of them died. ${ }^{9}$ Naik et al in a review of complications, due to PEG, mentioned that studies have reported complications as high as 70\%, which are mostly minor. Among the minor complications, peristomal infection is the commonest complication in most studies, being observed in up to $30 \%$ in some studies; peristomal leakage and inadvertent tube removal are other commonly encountered minor complications. Major complications, which are potentially life threatening, have been reported in up to two percent of cases and include, hemorrhage, peritonitis, and necrotizing fasciitis. ${ }^{10}$ In our series, 9 of 73 (12.3\%) of patients developed complications, of which one was fatal.

To conclude, although usually a safe procedure, PEG is known to have complications, occasionally fatal; hence, utmost caution must be exercised during placement, care must be taken to avoid complications, and must be managed appropriately at an early stage.

\section{Conflict of Interest}

None declared.

\section{References}

1 Gauderer MW, Ponsky JL, Izant RJ Jr. Gastrostomy without laparotomy: a percutaneous endoscopic technique. J Pediatr Surg 1980;15(6):872-875

2 Tang SJ, Wu R. Percutaneous endoscopic gastrostomy (pull method) and jejunal extension tube placement. Video J Encycl GI Endosc 2014;2(1):40-45

3 Lipp A, Lusardi G. Systemic antimicrobial prophylaxis for percutaneous endoscopic gastrostomy. Cochrane Database Syst Rev 2013; ( 11):CD005571

4 Banerjee S, Shen B, Baron TH, et al; ASGE STANDARDS OF PRACTICE COMMITTEE. Antibiotic prophylaxis for GI endoscopy. Gastrointest Endosc 2008;67(6):791-798

5 Magné N, Marcy PY, Foa C, et al. Comparison between nasogastric tube feeding and percutaneous fluoroscopic gastrostomy in advanced head and neck cancer patients. Eur Arch Otorhinolaryngol 2001;258(2):89-92

6 Sadasivan A, Faizal B, Kumar M. Nasogastric and percutaneous endoscopic gastrostomy tube use in advanced head and neck cancer patients: a comparative study. J Pain Palliat Care Pharmacother 2012;26(3):226-232

7 Enestvedt BK, Jorgensen J, Sedlack RE, et al. ASGE Training Committee 2013-2014. Endoscopic approaches to enteral feeding and nutrition core curriculum. Gastrointest Endosc 2014;80(1):34-41

8 Wiesen AJ, Sideridis K, Fernandes A, et al. True incidence and clinical significance of pneumoperitoneum after PEG placement: a prospective study. Gastrointest Endosc 2006;64(6):886-889

9 Mansoor H, Masood MA, Yusuf MA. Complications of percutaneous endoscopic gastrostomy tube insertion in cancer patients: a retrospective study. J Gastrointest Cancer 2014;45(4):452-459

10 Naik RP, Joshipura VP, Patel NR, Haribhakti SP. Complications of PEG-prevention and management. Trop Gastroenterol 2009;30(4):186-194 